\title{
Self-Reported Facial Pain in UK Biobank Study: Prevalence and Associated Factors
}

\author{
Tatiana V. Macfarlane', Marcus Beasley², Gary J. Macfarlane ${ }^{2}$ \\ ${ }^{1}$ University of Aberdeen Dental School, Aberdeen, United Kingdom. \\ ${ }^{2}$ Epidemiology Group, Aberdeen Pain Research Collaboration, University of Aberdeen, Aberdeen, Scotland, United \\ Kingdom.
}

\author{
Corresponding Author: \\ Tatiana Macfarlane \\ University of Aberdeen Dental School \\ Cornhill Road, Foresterhill, \\ Aberdeen, AB25 2ZR \\ United Kingdom \\ Phone: (+44) (0)1224 551585 \\ Email: Tatiana.Macfarlane@abdn.ac.uk
}

\begin{abstract}
Objectives: To determine the prevalence of facial pain and to examine the hypothesis that symptoms are associated with socio-demographic, dental, adverse psychological factors and pain elsewhere in the body.

Material and Methods: Cross-sectional population data were obtained from UK Biobank (www.ukbiobank.ac.uk/) study which was conducted in 2006 - 2010 and recruited over 500,000 people.

Results: The overall prevalence of facial pain (FP) was $1.9 \%$ (women $2.4 \%$, men $1.2 \%$ ) of which $48 \%$ was chronic. The highest prevalence was found in the $51-55$ age group (2.2\%) and the lowest in the 66 - 73 age group (1.4\%). There was a difference in prevalence by ethnicity $(0.8 \%$ and $2.7 \%$ in persons reporting themselves as Chinese and Mixed respectively). Prevalence of FP significantly associated with all measures of social class with the most deprived and on lowest income showing the highest prevalence $(2.5 \%$ and $2.4 \%$ respectively). FP was more common in individuals who rated themselves as extremely unhappy, had history of depression and reported sleep problems. Smoking associated with increase in reporting FP while alcohol consumption had inverse association. FP associated with history of painful gums, toothache and all types of regional pain.

Conclusions: This is the largest ever study to provide estimates of facial pain prevalence. It demonstrates unique features (lower prevalence than previously reported) and common features (more common in women) and confirms multifactorial aetiology of facial pain. Significant association with psychological distress and a strong relationship to pain elsewhere in the body suggests that aetiology is not specific to this regional pain.
\end{abstract}

Keywords: facial pain; orofacial pain; epidemiology; risk factors.

\author{
Accepted for publication: 29 August 2014 \\ To cite this article: \\ Macfarlane TV, Beasley M, Macfarlane GJ. Self-Reported Facial Pain in UK Biobank Study: Prevalence and Associated \\ Factors \\ J Oral Maxillofac Res 2014;5(3):e2 \\ URL: http://www.ejomr.org/JOMR/archives/2014/3/e2/v5n3e2ht.pdf \\ doi: $10.5037 /$ jomr.2014.5302
}




\section{INTRODUCTION}

Facial pain (FP) is a common symptom experienced by a quarter of the adult UK population [1]. Local mechanical factors, psychological factors and comorbidities have been determined to play a role in aetiology of FP [2, 3-7].

However a systematic review [5] showed a considerable variation in the prevalence of FP depending on its definition. The minimum prevalence was $1 \%$ (current cheek pain) and the maximum was $48 \%$ (current oral or facial pain) while the median prevalence of orofacial pain was $13 \%$ [ [5]. More recent studies showed the prevalence of $5 \%$ (jaw pain in the past month) [8], 10\% (temporomandubular [TMD]related pain) [9], 24\% (overall orofacial pain in the past 4 weeks excluding tooth sensitivity) [10] and $57 \%$ (current or episodic orofacial pain) [11]. The largest epidemiological study to date, conducted in a representative sample of 45,711 households of US civilian population [12], reported a prevalence of toothache $(12 \%)$, oral sores $(8 \%)$, jaw joint pain $(5 \%)$, face/check pain (1\%) and burning mouth $(1 \%)$.

The very large sample size of the UK Biobank study gives an opportunity to provide the most precise estimates of FP prevalence and the relationship of multiple factors with the report of symptoms. It allows for thorough assessment of confounding factors and the sample allows the exploration of interactions.

The purpose of the study was to determine the prevalence of FP in UK Biobank study and to examine the hypothesis that symptoms are associated with socio-demographic, dental, adverse psychological factors and pain elsewhere in the body.

\section{MATERIAL AND METHODS UK Biobank study}

The study aimed to recruit persons aged 40 - 69 years who were registered with general medical practitioner within the UK National Health Service (NHS). As it is estimated that over $95 \%$ of persons are so registered, this provides a suitable population sampling frame in the United Kingdom. Overall about 9.2 million invitations were issued to people living within about 25 miles of one of the 22 assessment centres in England, Scotland and Wales. In total the study recruited 503,325 people between 2006 and 2010, a participation rate of $5.5 \%$. Participants attended an assessment centre and completed questionnaires including items on lifestyle and environment.

The study has approval from the North West Multi- centre Research Ethics Committee. All participants gave informed consent. Detailed methods used by Biobank are described by Allen et al. [13] .

\section{Definition of facial pain (FP) and other regional pain}

Information on pain was collected by means of a touch screen questionnaire. Participants were asked "In the last month have you experienced any of the following that interfered with your usual activities?" They were then provided with a list: headache, facial pain, neck or shoulder pain, back pain, stomach or abdominal pain, hip pain, knee pain, pain all over the body. Participants were able to select more than one answer. For each site for which they answered positively, they were asked whether this pain had lasted at least three months, which we defined as chronic [14].

\section{Definition of exposure}

The determinants or exposures which we considered in relation to $\mathrm{FP}$ in this paper were demographic, socio-economic, psychological, sleep, behavioural factors and co-morbidities including dental factors.

\section{Socio-economic and demographic factors}

Specifically, information was available on gender, age and ethnic group. Ethnic group is classified as white, mixed ethnic group, Asian or Asian British, black or black British, Chinese, or other ethnic group. Information was also available on average total household income, current employment status, highest qualification achieved and Townsend deprivation index [15]. The Townsend deprivation index is continuous but has been categorised into ten groups using deciles of the overall distribution.

\section{Psychological factors}

Psychosocial factors we have used were self-defined happiness (In general how happy are you?) classified in six categories from extremely happy to extremely unhappy, work/job satisfaction (In general how satisfied are you with the WORK that you do?) classified in six categories from extremely happy to extremely unhappy, whether participants had ever been depressed for a whole week and the number of depressive episodes. Participants were also asked whether they had ever seen a general practitioner for "nerves, anxiety, tension or depression" and were asked about adverse life events such as illness, injury, bereavement or stress in the past 2 years. 


\section{Sleep}

Touchscreen question on sleep was "Do you have trouble falling asleep at night or do you wake up in the middle of the night?" (answer options were Never/ rarely, sometimes or usually) and referred to the past 4 weeks. Snoring was defined as a positive answer to the touchscreen question "Does your partner or a close relative or friend complain about your snoring?".

\section{Behavioural factors}

Participants were asked if they currently smoked and about their frequency of alcohol consumption (six point scale from daily or almost daily to never).

\section{Co-morbidities}

Participants rated their overall health in four categories as excellent, good, fair or poor. Questions on mouth and dental problems were related to the past year (mouth ulcers, painful gums, bleeding gums, loose teeth, toothache and dentures).

\section{Statistical analysis}

Prevalence rates of FP and other regional pains and of any chronic pain were calculated for respondents who had provided an answer to the pain question in the UK Biobank questionnaire. Directly standardised prevalence was calculated using UK population estimates in 2011 [16]. Further analysis was by Cox regression with results expressed as relative risks (RR) with $95 \%$ confidence intervals (CI). The RR is the ratio of FP probability in a group with risk factor divided by the FP probability in a group with no risk factor. RR can range from zero to infinity. In a study of two groups, an RR of 1 indicates that outcomes did not differ between the two groups, while an RR of 3 indicates that the group with a risk factor had a threefold greater probability than the group without it. Firstly, univariate analysis was conducted with adjustment for age group and gender. Further, multivariate (backward stepwise) models were built up separately for chronic and non-chronic FP aiming at creating the most parsimonious statistical model using the minimum of missing data. Missing data occurred when participants chose "prefer not to answer" option. We performed a complete data analysis excluding participants with missing data. We included variables which showed an association with FP in univariate analysis. For each final model, the number of factors was calculated for each participant and prevalence was investigated within each strata.
Analysis was conducted using Stata 13.0 statistical software [17].

\section{RESULTS}

Prevalence of facial pain by age, gender and ethnicity

In total 500,488 (99.4\%) participants in UK Biobank between 37 and 73 years old provided an answer for the question about pain they had experienced in the last month.

Of these 9,345 answered that they had FP, providing a crude prevalence of $1.87 \%$ (99\% CI $1.82 \%, 1.92 \%)$ and directly standardised prevalence $1.89 \%$ (99 CI $1.83 \%, 1.94 \%$ ), while 4,409 reported FP pain which was chronic, providing a prevalence of chronic FP of $0.88 \%(99 \%$ CI $0.85 \%, 0.92 \%)$ and directly standardised prevalence of FP $0.88 \%$ (99\% CI $0.84 \%$, $0.92 \%)$ (Table 1$)$.

FP prevalence peaked at $51-55$ years $(2.22 \%)$ and then decreased with older age to $1.4 \%$ in age group 66 - 73 years (Table 1). FP was more common in females than males $(2.41 \%$ vs. $1.22 \%$, RR $1.95,95 \%$ CI 1.86 , 2.04). There was no significant interaction between age and gender for facial pain or chronic facial pain. In comparison to persons who identified their ethnicity as "white" (prevalence of FP 1.86\%), persons identifying themselves as mixed ethnicity $(2.68 \%$, RR $1.27: 1.02,1.59)$ were significantly more likely to report pain (Table 1). Persons identifying themselves as of Chinese ethnicity were less likely to report FP $(0.83 \%$, RR 0.40: 0.23, 0.68). Similar results were observed for chronic FP.

\section{Facial pain reporting in relation to socio-economic factors}

The prevalence of FP was strongly related to various measures of social and economic status. The prevalence of FP decreased monotonically as income group increased from $2.45 \%$ amongst those with annual incomes less than $£ 18,000$ to $1.2 \%$ amongst those with income greater than $£ 100,000$ (Table 2). In relation to employment, FP was least common amongst those in paid employment $(1.72 \%)$ and those retired (1.69\%), while the highest prevalence was reported by those who were unable to work because of ill-health reported pain $(5.12 \%)$. Those who lived in most deprived areas reported the highest prevalence of FP (2.41\%). Similar results were observed for chronic FP. 
Table 1. Prevalence of facial pain by age, gender and ethnicity

\begin{tabular}{|c|c|c|c|c|c|}
\hline \multirow{2}{*}{ Factor } & \multirow{2}{*}{$\mathbf{N}$} & \multicolumn{2}{|c|}{$\begin{array}{l}\text { Facial pain } \\
(n=9,345)^{a}\end{array}$} & \multicolumn{2}{|c|}{$\begin{array}{l}\text { Chronic facial pain } \\
\quad(\mathrm{n}=4,409)^{\mathrm{b}}\end{array}$} \\
\hline & & $\begin{array}{l}\text { Prevalence } \\
(\%)\end{array}$ & $\operatorname{RR}(95 \% \mathrm{CI})^{\mathrm{c}}$ & $\begin{array}{l}\text { Prevalence } \\
\text { (\%) }\end{array}$ & $\operatorname{RR}(95 \% \mathrm{CI})^{\mathrm{c}}$ \\
\hline Overall & 500,488 & & & & \\
\hline Crude $(99 \% \mathrm{CI})$ & & $1.87(1.82,1.92)$ & - & $0.88(0.85,0.92)$ & - \\
\hline Adjusted (UK population) $(99 \% \mathrm{CI})$ & & $1.89(1.83,1.94)$ & - & $0.88(0.84,0.92)$ & - \\
\hline \multicolumn{6}{|l|}{ Age group (years) } \\
\hline $37-45$ & 64,143 & 2.09 & 1 & 0.97 & 1 \\
\hline $46-50$ & 67,409 & 2.2 & $1.04(0.97,1.21)$ & 1. & $1.02(0.92,1.13)$ \\
\hline $51-55$ & 78,609 & 2.22 & $1.04(0.97,1.12)$ & 1.06 & $1.07(0.97,1.19)$ \\
\hline $56-60$ & 98,013 & 1.87 & $0.89(0.83,0.95)$ & 0.9 & $0.92(0.83,1.02)$ \\
\hline $61-65$ & 118,811 & 1.61 & $0.77(0.72,0.83)$ & 0.76 & $0.78(0.71,0.87)$ \\
\hline $66-73$ & 73,503 & 1.4 & $0.68(0.63,0.74)$ & 0.66 & $0.7(0.62,0.79)$ \\
\hline \multicolumn{6}{|l|}{ Gender } \\
\hline Men & 228,150 & 1.22 & 1 & 0.53 & 1 \\
\hline Women & 272,338 & 2.41 & $1.95(1.86,2.04)$ & 1.18 & $2.21(2.07,2.36)$ \\
\hline \multicolumn{6}{|l|}{ Men by age group } \\
\hline $37-45$ & 29,417 & 1.44 & 1 & 0.55 & 1 \\
\hline $46-50$ & 29,747 & 1.48 & $1.03(0.9,1.17)$ & 0.62 & $1.13(0.91,1.39)$ \\
\hline $51-55$ & 34,013 & 1.54 & $1.07(0.95,1.22)$ & 0.68 & $1.23(1,1.5)$ \\
\hline $56-60$ & 43,450 & 1.22 & $0.85(0.75,0.97)$ & 0.54 & $0.97(0.8,1.19)$ \\
\hline $61-65$ & 55,163 & 1.03 & $0.72(0.63,0.81)$ & 0.45 & $0.82(0.67,0.99)$ \\
\hline $66-73$ & 36,360 & 0.84 & $0.58(0.5,0.67)$ & 0.4 & $0.72(0.58,0.91)$ \\
\hline \multicolumn{6}{|l|}{ Women by age group } \\
\hline $37-45$ & 34,726 & 2.65 & 1 & 1.33 & 1 \\
\hline $46-50$ & 37,662 & 2.78 & $1.05(0.96,1.15)$ & 1.31 & $0.98(0.87,1.12)$ \\
\hline $51-55$ & 44,596 & 2.73 & $1.03(0.95,1.12)$ & 1.35 & $1.02(0.9,1.15)$ \\
\hline $56-60$ & 54,563 & 2.39 & $0.9(0.83,0.98)$ & 1.2 & $0.9(0.8,1.02)$ \\
\hline $61-65$ & 63,648 & 2.11 & $0.8(0.73,0.87)$ & 1.03 & $0.77(0.69,0.87)$ \\
\hline $66-73$ & 37,143 & 1.94 & $0.73(0.67,0.81)$ & 0.92 & $0.69(0.6,0.8)$ \\
\hline \multicolumn{6}{|l|}{ Ethnicity } \\
\hline White & 472,013 & 1.86 & 1 & 0.88 & 1 \\
\hline Mixed & 2,948 & 2.68 & $1.27(1.02,1.59)$ & 1.56 & $1.57(1.17,2.1)$ \\
\hline Asian or Asian British & 9,745 & 1.6 & $0.86(0.73,1.01)$ & 0.74 & $0.85(0.67,1.07)$ \\
\hline Black or Black British & 8,001 & 2.1 & $1.03(0.88,1.2)$ & 0.91 & $0.95(0.75,1.19)$ \\
\hline Chinese & 1,558 & 0.83 & $0.4(0.23,0.68)$ & 0.39 & $0.4(0.17,0.86)$ \\
\hline Other ethnic group & 4,507 & 1.91 & $0.95(0.76,1.17)$ & 0.8 & $0.96(0.61,1.17)$ \\
\hline No information & 1,716 & & & & \\
\hline
\end{tabular}

aFacial pain that interfered with participants' usual activities.

${ }^{b}$ Chronic facial pain was defined as facial pains for more than 3 months. 132 participants did not answer the question about chronicity and were excluded.

${ }^{\mathrm{c}}$ Adjusted for age group and gender.

$\mathrm{RR}=$ relative risks. 
Table 2. Prevalence of facial pain by socio-economic factors

\begin{tabular}{|c|c|c|c|c|c|}
\hline \multirow{2}{*}{ Factor } & \multirow{2}{*}{$\mathbf{N}$} & \multicolumn{2}{|c|}{$\begin{array}{l}\text { Facial pain } \\
(\mathrm{n}=9,345)^{\mathrm{a}}\end{array}$} & \multicolumn{2}{|c|}{$\begin{array}{l}\text { Chronic facial pain } \\
\quad(\mathrm{n}=4,409)^{\mathrm{b}}\end{array}$} \\
\hline & & $\begin{array}{c}\text { Prevalence } \\
(\%)\end{array}$ & $\operatorname{RR}(95 \% \mathrm{CI})^{\mathrm{c}}$ & $\begin{array}{c}\text { Prevalence } \\
(\%)\end{array}$ & $\operatorname{RR}(95 \% \mathrm{CI})^{\mathrm{c}}$ \\
\hline \multicolumn{6}{|l|}{ Average total household income before tax (£) } \\
\hline$<18,000$ & 96,895 & 2.45 & $1^{\mathrm{d}}$ & 1.25 & $1^{\mathrm{d}}$ \\
\hline $18,000-$ & 108,048 & 1.84 & $0.73(0.68,0.77)$ & 0.84 & $0.65(0.6,0.71)$ \\
\hline $31,000-$ & 110,715 & 1.68 & $0.62(0.58,0.66)$ & 0.76 & $0.55(0.5,0.6)$ \\
\hline $52,000-$ & 86,245 & 1.55 & $0.55(0.51,0.59)$ & 0.7 & $0.49(0.44,0.54)$ \\
\hline $100,000+$ & 22,930 & 1.2 & $0.43(0.37,0.48)$ & 0.54 & $0.38(0.31,0.46)$ \\
\hline No information & 75,655 & & & & \\
\hline \multicolumn{6}{|l|}{ Current employment status } \\
\hline In paid employment or self-employed & 286,794 & 1.72 & 1 & 0.76 & 1 \\
\hline Retired & 166,592 & 1.69 & $1.27(1.19,1.35)$ & 0.82 & $1.44(1.31,1.58)$ \\
\hline Looking after home and/or family & 13,798 & 2.64 & $1.21(1.09,1.35)$ & 1.09 & $1.09(0.93,1.29)$ \\
\hline Unable to work because of sickness or disability & 16,734 & 5.12 & $3.18(2.95,3.42)$ & 3.18 & $4.51(4.1,4.96)$ \\
\hline Unemployed & 8,213 & 2.18 & $1.4(1.21,1.63)$ & 1.05 & $1.56(1.25,1.93)$ \\
\hline Doing unpaid or voluntary work & 2,313 & 2.68 & $1.5(1.16,1.92)$ & 1.12 & $1.41(0.95,2.07)$ \\
\hline Student & 1,337 & 2.69 & $1.35(0.97,1.87)$ & 1.2 & $1.33(0.81,2.18)$ \\
\hline No information & 4,707 & & & & \\
\hline \multicolumn{6}{|l|}{ Highest qualification achieved } \\
\hline University or college degree & 161,028 & 1.71 & 1 & 0.8 & 1 \\
\hline A/AS level & 55,267 & 1.94 & $1.1(1.02,1.18)$ & 0.85 & $1.03(0.92,1.14)$ \\
\hline O level /GCSEs & 105,062 & 1.96 & $1.13(1.06,1.19)$ & 0.95 & $1.16(1.08,1.26)$ \\
\hline CSEs & 26,828 & 2.26 & $1.24(1.13,1.35)$ & 0.96 & $1.13(0.98,1.29)$ \\
\hline $\mathrm{NVQ} / \mathrm{HND} / \mathrm{HNC}$ & 32,661 & 1.83 & $1.23(1.13,1.35)$ & 0.84 & $1.24(1.08,1.41)$ \\
\hline Other professional qualifications & 25,765 & 1.78 & $1.08(0.98,1.19)$ & 0.9 & $1.15(1.00,1.33)$ \\
\hline None of the above & 84,877 & 1.89 & $1.24(1.16,1.32)$ & 0.95 & $1.34(1.22,1.47)$ \\
\hline No information & 9,000 & & & & \\
\hline \multicolumn{6}{|l|}{ Townsend deprivation index } \\
\hline 1 Least deprived & 49,812 & 1.62 & $1^{\mathrm{d}}$ & 0.68 & $1^{\mathrm{d}}$ \\
\hline 2 & 50,139 & 1.65 & $1.02(0.93,1.12)$ & 0.74 & $1.11(0.95,1.28)$ \\
\hline 3 & 49,975 & 1.71 & $1.06(0.96,1.17)$ & 0.8 & $1.18(1.02,1.37)$ \\
\hline 4 & 49,894 & 1.74 & $1.07(0.97,1.18)$ & 0.78 & $1.15(0.99,1.33)$ \\
\hline 5 & 50,043 & 1.74 & $1.07(0.97,1.18)$ & 0.81 & $1.19(1.03,1.38)$ \\
\hline 6 & 50,011 & 1.82 & $1.11(1.01,1.22)$ & 0.84 & $1.23(1.06,1.42)$ \\
\hline 7 & 50,011 & 1.94 & $1.18(1.07,1.29)$ & 0.93 & $1.35(1.18,1.56)$ \\
\hline 8 & 49,987 & 1.87 & $1.13(1.03,1.24)$ & 0.96 & $1.4(1.21,1.6)$ \\
\hline 9 & 49,981 & 2.16 & $1.31(1.19,1.43)$ & 1.05 & $1.53(1.33,1.75)$ \\
\hline 10 Most deprived & 50,012 & 2.41 & $1.47(1.35,1.61)$ & 1.21 & $1.79(1.57,2.04)$ \\
\hline No information & 623 & & & & \\
\hline
\end{tabular}

${ }^{a}$ Facial pain that interfered with participants' usual activities.

${ }^{b}$ Chronic facial pain was defined as facial pains for more than 3 months. 132 participants did not answer the question about chronicity and were excluded.

${ }^{\mathrm{c}}$ Adjusted for age group and gender.

${ }^{\mathrm{d}}$ Test for trend $\mathrm{P}<0.001$.

$\mathrm{RR}=$ relative risks. 
Facial pain reporting in relation to psychosocial factors

The prevalence of FP was strongly related to various measures of psychological distress. Those who had consulted a GP for "nerves, anxiety, tension or depression" $(3.02 \%$ vs. $1.27 \%$; RR 2.16: $2.08,2.25)$ had an excess risk of reporting
FP and the likelihood of reporting FP increased strongly with the reported number of episodes of depression (No episodes: 0.93\%; 1 episode: 1.37\%, 2 - 3 episodes $1.75 \%$; $>3$ episodes $2.86 \%$ ) (Table 3 ). Similarly there was a monotonic increase in the prevalence of FP reporting according to how unhappy respondents were (extremely happy $0.95 \%$ to extremely unhappy 6\% RR 6.12: $3.89,9.61)$.

Table 3. Prevalence of facial pain by psychological factors

\begin{tabular}{|c|c|c|c|c|c|}
\hline \multirow[t]{2}{*}{ Factor } & \multirow[t]{2}{*}{$\mathbf{N}$} & \multicolumn{2}{|c|}{$\begin{array}{l}\text { Facial pain } \\
(\mathrm{n}=9,345)^{\mathrm{a}}\end{array}$} & \multicolumn{2}{|c|}{$\begin{array}{l}\text { Chronic facial pain } \\
\qquad\left(\mathrm{n}=\mathbf{4 , 4 0 9 ) ^ { \mathrm { b } }}\right.\end{array}$} \\
\hline & & Prevalence (\%) & $\operatorname{RR}(95 \% \mathrm{CI})^{\mathrm{c}}$ & Prevalence (\%) & $\operatorname{RR}(95 \% \mathrm{CI})^{\mathrm{c}}$ \\
\hline \multicolumn{6}{|l|}{ Happiness } \\
\hline Extremely happy & 9,236 & 0.95 & $1^{\mathrm{d}}$ & 0.37 & $1^{\mathrm{d}}$ \\
\hline Very happy & 65,186 & 1.13 & $1.14(0.92,1.43)$ & 0.55 & $1.43(1,2.03)$ \\
\hline Moderately happy & 88,421 & 1.74 & $1.72(1.38,2.13)$ & 0.86 & $2.17(1.54,3.06)$ \\
\hline Moderately unhappy & 6,449 & 3.02 & $3.01(2.34,3.87)$ & 1.61 & $4.15(2.82,6.12)$ \\
\hline Very unhappy & 1,278 & 4.54 & $4.48(3.22,6.25)$ & 2.9 & $7.44(4.67,11.87)$ \\
\hline Extremely unhappy & 400 & 6 & $6.12(3.89,9.61)$ & 4 & $10.64(5.87,19.3)$ \\
\hline No information & 329,518 & & & & \\
\hline \multicolumn{6}{|l|}{ Work/job satisfaction } \\
\hline Extremely happy & 10,067 & 1.17 & 1 & 0.62 & 1 \\
\hline Very happy & 43,166 & 1.21 & $1(0.82,1.23)$ & 0.55 & $0.87(0.66,1.15)$ \\
\hline Moderately happy & 55,120 & 1.47 & $1.19(0.98,1.44)$ & 0.71 & $1.09(0.83,1.42)$ \\
\hline Moderately unhappy & 8,544 & 2.07 & $1.73(1.37,2.19)$ & 0.98 & $1.58(1.14,2.51)$ \\
\hline Very unhappy & 2,219 & 2.43 & $2.08(1.5,2.87)$ & 1.4 & $2.34(1.51,3.6)$ \\
\hline Extremely unhappy & 1,012 & 3.75 & $3.11(2.15,4.48)$ & 1.88 & $3.00(1.79,5.03)$ \\
\hline Not currently employed & 50,703 & 1.8 & & 0.94 & \\
\hline No information & 329,657 & & & & \\
\hline \multicolumn{6}{|c|}{ Ever depressed for a whole week } \\
\hline No & 78,638 & 0.93 & 1 & 0.44 & 1 \\
\hline Yes & 89,256 & 2.09 & $2.05(1.88,2.23)$ & 1.05 & $2.16(1.91,2.44)$ \\
\hline No information & 332,594 & & & & \\
\hline \multicolumn{6}{|c|}{ Number of depressive episodes } \\
\hline 0 & 78,638 & 0.93 & $1^{\mathrm{d}}$ & 0.44 & $1^{\mathrm{d}}$ \\
\hline 1 & 22,026 & 1.37 & $1.37(1.29,1.57)$ & 0.64 & $1.36(1.12,1.65)$ \\
\hline $2-3$ & 23,577 & 1.75 & $1.7(1.5,1.92)$ & 0.78 & $1.59(1.33,1.9)$ \\
\hline $4+$ & 18,637 & 2.86 & $2.81(2.51,3.15)$ & 1.51 & $3.1(2.64,3.63)$ \\
\hline No information & 357,610 & & & & \\
\hline \multicolumn{6}{|c|}{ Seen doctor (GP) for nerves, anxiety, tension or depression } \\
\hline No & 327,541 & 1.27 & 1 & 0.56 & 1 \\
\hline Yes & 169,180 & 3.02 & $2.16(2.08,2.25)$ & 1.49 & $2.37(2.23,2.51)$ \\
\hline No information & 3,767 & & & & \\
\hline \multicolumn{6}{|c|}{ Number of adverse life events in past 2 years } \\
\hline 0 & 271,981 & 1.46 & $1^{\mathrm{d}}$ & 0.66 & $1^{\mathrm{d}}$ \\
\hline 1 & 162,418 & 1.99 & $1.32(1.26,1.38)$ & 0.93 & $1.37(1.28,1.47)$ \\
\hline 2 & 47,353 & 2.89 & $1.85(1.74,1.97)$ & 1.41 & $2(1.83,2.18)$ \\
\hline $3+$ & 11,862 & 5.09 & $3.16(2.9,3.44)$ & 2.92 & $4.01(3.57,4.51)$ \\
\hline No information & 6,874 & & & & \\
\hline
\end{tabular}

aFacial pain that interfered with participants' usual activities.

${ }^{\mathrm{b}}$ Chronic facial pain was defined as facial pains for more than 3 months. 132 participants did not answer the question about chronicity and were excluded.

${ }^{\mathrm{c}}$ Adjusted for age group and gender.

dTest for trend $\mathrm{P}<0.001$.

$\mathrm{RR}=$ relative risks 
The same relationship was found when respondents, who were employed, were asked about how satisfied they were with their job (extremely satisfied $1.17 \%$ to extremely dissatisfied $3.75 \%$ RR $3.11: 2.15,4.48$ ). Finally in relation to life events in the past two years, there was an increase in FP prevalence with number of adverse events (1.46\% with 0 events, up to $5.09 \%$ for 3 or more events RR 3.16: 2.90, 3.44). Similar results were observed for chronic FP.

Facial pain reporting in relation to general health and sleep

Poor general health was associated with FP and chronic FP (RR 8.2; 95\% CI 7.44, 9.03 and 15.29
95\% CI 13.14, 17.79, respectively). Participants reporting sleep problems and snoring were more likely to report FP (RR 2.19; 95\% CI 2.05, 2.33 and $1.2395 \% \mathrm{CI} 1.17,1.28)$ and chronic FP (RR $2.59 ; 95 \%$ CI $2.37,2.85$ and $1.295 \%$ CI $1.13,1.28)$ (Table 4).

\section{Facial pain reporting in relation to smoking and alcohol consumption}

Smoking was associated with an increased risk of FP (RR $1.495 \%$ CI 1.33, 1.51) while alcohol had a protective effect (RR $0.5995 \%$ CI $0.54,0.63$ ) for those who consumed alcohol daily or almost daily compared to never drinkers (Table 4).

Table 4. Prevalence of facial pain by sleep problems, smoking and alcohol consumption

\begin{tabular}{|c|c|c|c|c|c|}
\hline \multirow[t]{2}{*}{ Factor } & \multirow[t]{2}{*}{$\mathbf{N}$} & \multicolumn{2}{|c|}{$\begin{array}{l}\text { Facial pain } \\
(\mathrm{n}=9,345)^{\mathrm{a}}\end{array}$} & \multicolumn{2}{|c|}{$\begin{array}{l}\text { Chronic facial pain } \\
(\mathrm{n}=4,409)^{\mathrm{b}}\end{array}$} \\
\hline & & Prevalence (\%) & $\operatorname{RR}(95 \% \mathrm{CI})^{\mathrm{c}}$ & Prevalence (\%) & $\operatorname{RR}(95 \% \mathrm{CI})^{\mathrm{c}}$ \\
\hline \multicolumn{6}{|l|}{ Overall health } \\
\hline Excellent & 81,835 & 0.74 & $1^{\mathrm{d}}$ & 0.26 & $1^{\mathrm{d}}$ \\
\hline Good & 288,592 & 1.5 & $2.06(1.89,2.24)$ & 0.61 & $2.41(2.09,2.78)$ \\
\hline Fair & 105,067 & 2.89 & $4.15(3.8,4.52)$ & 1.5 & $6.24(5.4,7.2)$ \\
\hline Poor & 22,714 & 5.68 & $8.20(7.44,9.03)$ & 3.64 & $15.29(13.14,17.79)$ \\
\hline No information & 2,280 & & & & \\
\hline \multicolumn{6}{|l|}{ Sleep problems } \\
\hline Never/rarely & 120,584 & 1.16 & $1^{\mathrm{d}}$ & 0.5 & $1^{\mathrm{d}}$ \\
\hline Sometimes & 238,351 & 1.73 & $1.42(1.33,1.51)$ & 0.75 & $1.44(1.31,1.58)$ \\
\hline Usually & 141,134 & 2.71 & $2.19(2.05,2.33)$ & 1.41 & $2.59(2.37,2.85)$ \\
\hline No information & 419 & & & & \\
\hline \multicolumn{6}{|l|}{ Snoring } \\
\hline No & 291,626 & 1.8 & 1 & 0.86 & 1 \\
\hline Yes & 173,100 & 1.91 & $1.23(1.17,1.28)$ & 0.88 & $1.20(1.13,1.28)$ \\
\hline No information & 35,762 & & & & \\
\hline \multicolumn{6}{|l|}{ Smoking } \\
\hline No & 447,389 & 1.81 & 1 & 0.85 & 1 \\
\hline Only occasionally & 13,700 & 1.9 & $1.11(0.98,1.25)$ & 0.8 & $1(0.83,1.22)$ \\
\hline Yes, on most or all days & 39,102 & 2.52 & $1.42(1.33,1.51)$ & 1.22 & $1.47(1.34,1.62)$ \\
\hline No information & 297 & & & & \\
\hline \multicolumn{6}{|l|}{ Alcohol intake frequency } \\
\hline Never & 40,409 & 2.74 & 1 & 1.53 & 1 \\
\hline Special occasions only & 57,805 & 2.63 & $0.92(0.85,0.99)$ & 1.34 & $0.84(0.75,0.93)$ \\
\hline One to three times a month & 55,767 & 2.33 & $0.83(0.76,0.9)$ & 1.11 & $0.71(0.63,0.79)$ \\
\hline Once or twice a week & 129,069 & 1.73 & $0.65(0.61,0.7)$ & 0.8 & $0.55(0.49,0.6)$ \\
\hline Three or four times a week & 115,325 & 1.49 & $0.58(0.54,0.63)$ & 0.63 & $0.45(0.4,0.5)$ \\
\hline Daily or almost daily & 101,691 & 1.42 & $0.59(0.54,0.63)$ & 0.62 & $0.47(0.42,0.52)$ \\
\hline No information & 422 & & & & \\
\hline
\end{tabular}

${ }^{a}$ Facial pain that interfered with participants' usual activities.

${ }^{\mathrm{b}}$ Chronic facial pain was defined as facial pains for more than 3 months. 132 participants did not answer the question about chronicity and were excluded.

${ }^{\mathrm{c}}$ Adjusted for age group and gender.

${ }^{\mathrm{d}}$ Test for trend $\mathrm{P}<0.001$.

$\mathrm{RR}=$ relative risks. 


\section{Facial pain reporting in relation to dental factors}

FP was more common in individuals with history in the past year of mouth ulcers $(2.0195 \%$ CI 1.90 , 2.11), painful gums $(4.2795 \%$ CI $4.01,4.54)$, bleeding gums $(1.4595 \%$ CI 1.37, 1.52), loose teeth $(1.6395 \%$ CI $1.5,1.77)$, toothache $(4.2495 \%$ CI 4.01 , $4.48)$ and those wearing dentures $(1.1595 \%$ CI 1.08 , 1.21) (Table 5).

Facial pain reporting in relation to other types of pain

Reporting of FP was particularly associated with headache (RR 6.01, 95\% CI 5.76, 6.27), but was also significantly related to all other types of pain (Table 6).

\section{Multivariate model}

The final multivariate model for FP included 16 variables which were associated with increased risk: age (51 - 55 years), female gender, ethnicity (white or mixed), employment (retired, unable to work, doing unpaid work or student), adverse life events
( 2 or more), mouth ulcers, painful gums, toothache, headache, neck or shoulder pain, back pain, stomach pain, hip pain, knee pain, sleep problems, alcohol consumption (never) and general health (poor). Data for all the above variables were available for 480,967 participants. Table 7 shows monotonic increase in prevalence of FP with increase of the number of factors from the model from $0.33 \%$ on those reporting none or any 1 factor to $27.47 \%$ among those reporting 12 or more factors

The final multivariate model for chronic FP included 19 variables: age (51 - 55 years), female gender, ethnicity (white or mixed), employment (retired, unable to work, doing unpaid work or student), adverse life events ( 2 or more), mouth ulcers, painful gums, bleeding gums, toothache, denture, headache, neck or shoulder pain, back pain, stomach pain, hip pain, knee pain, sleep problems, alcohol consumption (never) and general health (poor). Data were available for 482,663 participants. Table 7 shows monotonic increase in prevalence of chronic FP with increase of the number of factors from the model from $0.11 \%$ on those reporting none or any 1 factor to $10.87 \%$ among those reporting 12 or more factors.

Table 5. Prevalence of facial pain by dental factors

\begin{tabular}{|c|c|c|c|c|c|}
\hline \multirow[t]{2}{*}{ Factor } & \multirow[t]{2}{*}{$\mathbf{N}$} & \multicolumn{2}{|c|}{$\begin{array}{l}\text { Facial pain } \\
(\mathrm{n}=9,345)^{\mathrm{a}}\end{array}$} & \multicolumn{2}{|c|}{$\begin{array}{c}\text { Chronic facial pain } \\
(\mathrm{n}=4,409)^{\mathrm{b}}\end{array}$} \\
\hline & & Prevalence (\%) & $\operatorname{RR}(95 \% \mathrm{CI})^{\mathrm{c}}$ & Prevalence (\%) & $\operatorname{RR}(95 \% \mathrm{CI})^{\mathrm{c}}$ \\
\hline \multicolumn{6}{|l|}{ Mouth ulcers } \\
\hline No & 445,154 & 1.68 & 1 & 0.78 & 1 \\
\hline Yes & 50,183 & 3.51 & $2.01(1.9,2.11)$ & 1.76 & $2.15(2,2.32)$ \\
\hline No information & 5,151 & & & & \\
\hline \multicolumn{6}{|l|}{ Painful gums } \\
\hline No & 480,133 & 1.68 & 1 & 0.79 & 1 \\
\hline Yes & 15,204 & 7.62 & $4.27(4.01,4.54)$ & 3.89 & $4.62(4.24,5.04)$ \\
\hline No information & 5,151 & & & & \\
\hline \multicolumn{6}{|l|}{ Bleeding gums } \\
\hline No & 429,060 & 1.73 & 1 & 0.82 & 1 \\
\hline Yes & 66,277 & 2.74 & $1.45(1.37,1.52)$ & 1.27 & $1.4(1.30,1.51)$ \\
\hline No information & 5,151 & & & & \\
\hline \multicolumn{6}{|l|}{ Loose teeth } \\
\hline No & 473,694 & 1.82 & 1 & 0.86 & 1 \\
\hline Yes & 21,643 & 2.86 & $1.63(1.5,1.77)$ & 1.28 & $1.54(1.37,1.74)$ \\
\hline No information & 5,151 & & & & \\
\hline \multicolumn{6}{|l|}{ Toothache } \\
\hline No & 473,171 & 1.63 & 1 & 0.77 & 1 \\
\hline Yes & 22,166 & 6.88 & $4.24(4.01,4.48)$ & 3.22 & $4.22(3.89,4.58)$ \\
\hline No information & 5,151 & & & & \\
\hline \multicolumn{6}{|l|}{ Dentures } \\
\hline No & 412,333 & 1.87 & 1 & 0.88 & 1 \\
\hline Yes & 83,004 & 1.86 & $1.15(1.08,1.21)$ & 0.9 & $1.17(1.08,1.27)$ \\
\hline No information & 5,151 & & & & \\
\hline
\end{tabular}

aFacial pain that interfered with participants' usual activities.

${ }^{\mathrm{b}}$ Chronic facial pain was defined as facial pains for more than 3 months. 132 participants did not answer the question about chronicity and were excluded.

${ }^{\mathrm{c} A d j u s t e d ~ f o r ~ a g e ~ g r o u p ~ a n d ~ g e n d e r . ~}$

$\mathrm{RR}=$ relative risks 
Table 6. Prevalence of facial pain by other types of pain

\begin{tabular}{|c|c|c|c|c|c|}
\hline \multirow[t]{2}{*}{ Factor } & \multirow[t]{2}{*}{$\mathbf{N}$} & \multicolumn{2}{|c|}{$\begin{array}{l}\text { Facial pain } \\
(\mathrm{n}=9,345)^{\mathrm{a}}\end{array}$} & \multicolumn{2}{|c|}{$\begin{array}{l}\text { Chronic facial pain } \\
\qquad(\mathrm{n}=4,409)^{\mathrm{b}}\end{array}$} \\
\hline & & Prevalence (\%) & $\operatorname{RR}(95 \% \mathrm{CI})^{\mathrm{c}}$ & Prevalence (\%) & $\operatorname{RR}(95 \% \mathrm{CI})^{\mathrm{c}}$ \\
\hline \multicolumn{6}{|c|}{ Headache } \\
\hline No & 397,478 & 0.89 & 1 & 0.38 & 1 \\
\hline Yes & 103,010 & 5.64 & $6.01(5.76,6.27)$ & 2.8 & $6.87(6.45,7.32)$ \\
\hline \multicolumn{6}{|c|}{ Neck or shoulder pain } \\
\hline No & 383,447 & 1.18 & 1 & 0.5 & 1 \\
\hline Yes & 117,041 & 4.1 & $3.39(3.25,3.53)$ & 2.12 & $4.12(3.88,4.37)$ \\
\hline \multicolumn{6}{|c|}{ Back pain } \\
\hline No & 370,352 & 1.42 & 1 & 0.62 & 1 \\
\hline Yes & 130,136 & 3.13 & $2.23(2.14,2.32)$ & 1.62 & $2.67(2.51,2.83)$ \\
\hline \multicolumn{6}{|c|}{ Stomach pain } \\
\hline No & 456,483 & 1.51 & 1 & 0.69 & 1 \\
\hline Yes & 44,005 & 5.55 & $3.44(3.29,3.61)$ & 2.91 & $3.97(3.72,4.24)$ \\
\hline \multicolumn{6}{|l|}{ Hip pain } \\
\hline No & 444,177 & 1.6 & 1 & 0.73 & 1 \\
\hline Yes & 56,311 & 3.97 & $2.44(2.33,2.56)$ & 2.1 & $2.82(2.64,3.02)$ \\
\hline \multicolumn{6}{|c|}{ Knee pain } \\
\hline No & 392,192 & 1.55 & 1 & 0.7 & 1 \\
\hline Yes & 108,164 & 3.02 & $2.03(1.94,2.11)$ & 1.55 & $2.31(2.17,2.45)$ \\
\hline
\end{tabular}

aFacial pain that interfered with participants' usual activities.

${ }^{\mathrm{b}}$ Chronic facial pain was defined as facial pains for more than 3 months. 132 participants did not answer the question about chronicity and were excluded.

${ }^{\mathrm{c}}$ Adjusted for age group and gender.

$\mathrm{RR}=$ relative risks

Table 7. Prevalence of facial pain by number of factors from the final multivariate model

\begin{tabular}{c|c|c|c|c}
\hline \multirow{2}{*}{ Number of factors reported } & \multicolumn{2}{|c|}{ Facial pain $^{\mathbf{e}}$} & \multicolumn{2}{c}{ Chronic facial pain $^{\mathbf{f}}$} \\
\cline { 2 - 5 } & $\mathbf{N}$ & Prevalence (\%) $^{\text {N }}$ & $\mathbf{N}$ & Prevalence (\%) $^{\text {(\%, }}$ \\
\hline $0-1$ & 28,906 & 0.33 & 23,413 & 0.11 \\
\hline 2 & 75,060 & 0.43 & 63,702 & 0.15 \\
\hline 3 & 102,169 & 0.67 & 93,186 & 0.24 \\
\hline 4 & 94,838 & 1.12 & 93,222 & 0.39 \\
\hline 5 & 69,840 & 1.81 & 74,493 & 0.61 \\
\hline 6 & 46,224 & 2.78 & 52,291 & 1.11 \\
\hline 7 & 28,468 & 4.08 & 33,684 & 1.64 \\
\hline 8 & 16,651 & 5.59 & 20,600 & 2.23 \\
\hline 9 & 9,256 & 7.84 & 12,121 & 3.67 \\
\hline 10 & 4,984 & 10.39 & 6,955 & 4.83 \\
\hline 11 & 2,474 & 14.35 & 3,647 & 6.94 \\
\hline 12 & 1,227 & 18.01 & 3,541 & 10.87 \\
\hline $13+$ & 870 & 27.47 & & \\
\hline
\end{tabular}

${ }^{\mathrm{e}}$ Model included: Age (51 - 55 years), female gender, ethnicity (white or mixed), employment (retired, unable to work, doing unpaid work or student), adverse life events ( 2 or more), mouth ulcers, painful gums, toothache, headache, neck or shoulder pain, back pain, stomach pain, hip pain, knee pain, sleep problems, alcohol consumption (never), general health (poor). Data were available for 480,967 participants. fModel included: Age (51 - 55 years), female gender, ethnicity (white or mixed), employment (retired, unable to work, doing unpaid work or student), adverse life events ( 2 or more), mouth ulcers, painful gums, bleeding gums, toothache, denture, headache, neck or shoulder pain, back pain, stomach pain, hip pain, knee pain, sleep problems, alcohol consumption (never) and general health (poor). Data were available for 480,835 participants. 


\section{DISCUSSION}

UK Biobank study is the largest ever study to provide estimates of FP prevalence. It demonstrates unique features (lower prevalence than previously reported) and common features (more common in women) and confirms multifactorial aetiology of FP. Significant association with psychological distress and a strong relationship to pain elsewhere in the body suggests that aetiology is not specific to this regional pain.

However the participation rate in this study was low which can result in biased prevalence estimates. We have presented both crude and adjusted (UK population) figures for the prevalence which were not very different. Another aspect which may influenced the results is the fact that the main question on various types of pain did not permit to specify any additional type of pain for those who reported widespread body pain.

The lower prevalence of FP demonstrated in this study could have been due to the way the question on FP was asked. For example, in the study by Macfarlane et al. [1] FP was measured as any of the following: Pain in the jaw joint/s; Pain in area just in front of the ear/s; Pain in or around the eyes; Pain when opening the mouth wide; Shooting pains in the face or cheeks; Pain in the jaw joint when chewing food; Pain in and around the temples; Tenderness of muscles at the side of the face; A prolonged burning sensation in the tongue or other parts of the mouth. Is it also possible that the population of the participants have increased painful comorbidities of which a rather small proportion reports FP.

The UK Biobank study used questionnaire based on a review of questionnaires previously used in epidemiological studies which was conducted in order to identify appropriate questions to quantify exposures, and involved wide consultation with international experts in each area of interest [18].

The UK Biobank study can be assessed as of high quality using instrument for assessing the quality of prevalence studies [19] because it is very large and therefore has sufficient statistical power, conducted according to ethical principles and scientific methodology using valid survey instruments. Total of 500,488 participants completed the pain question of 503,325 which is an excellent response rate of $99.4 \%$ for this question.

Previous population studies have found a higher prevalence of FP symptoms among younger people $[\underline{15}, \underline{20-22}, \underline{25}]$. The lowest prevalence of orofacial pain was seen in individuals aged more than 60 years. However, other studies have reported alternative relationships: a bell-shape age distribution [23], increasing with age [24] and no change with age [11]. While the current study found the lowest prevalence of FP in $66-73$ age group it did not include younger people (under 37 years) in the study sample. The overall prevalence is lower than previously reported may be also due to the age group considered in this study which does not include younger people.

We found higher prevalence of FP in women compared to men, similar to previous studies $[\underline{1}, \underline{8}, \underline{9}, \underline{21}]$.

It is difficult to compare the findings between studies examining different facets of social status. Macfarlane et al. [1, $\underline{6}$ ] did not find a relationship overall in either the cross-sectional or case-control study between education and orofacial pain, including TMD. When employment status was considered, a moderate increase in risk was found for housewives/ husbands, working part-time, and students; however, these associations disappeared after adjustment for age and gender. The only significant increase in risk was observed, unsurprisingly, for the group of people who reported not working because of ill health or disability [1]. Lower socio-economic status (SES) was associated with reporting orofacial pain and the effects of SES on orofacial pain appear to have a sexdifferentiated effect [25].

Our results show variation in prevalence by ethnicity with the lowest prevalence of FP among the Chinese. Lipton et al. [12] reported that whites had the greatest estimated prevalence rate for FP and jaw joint pain in the US population.

Cross-sectional $[2,9,26]$ and case-control $[6,27]$ studies showed an association between different measures of psychological distress and orofacial pain. However, a cohort study which controlled for confounding factors did not find an increase in risk of onset of TMD pain in three years [28], but found strong associations between signs of depression at baseline and first onset rates of other pain such as headaches and chest pain. A large population-based retrospective cohort study from Taiwan showed an increased risk of developing TMD in patients with depression compared to those without depression (hazards ratio [HR] $=2.2$ ) [29].

Both the cross-sectional and case-control studies noted above indicated a strong doseresponse relationship with increasing total sleep

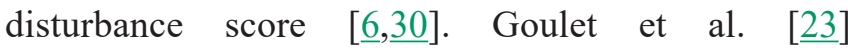
reported that participants with sleep problems were twice as likely to report jaw pain. However, observational studies are unable to establish the temporal relationship with sleep disturbance as it could precede or be a consequence of pain. 
However, Smythe and Moldofsky [31] reported that disturbance of sleep itself increases the likelihood of subsequently reporting pain symptoms. The symptoms disappeared with subsequent uninterrupted sleep. Sanders et al. [32] investigated the relationship between obstructive sleep apnea (OSA) and TMD by using data from the OPPERA prospective cohort study and the OPPERA case-control study of chronic TMD. Both studies supported a significant association of OSA symptoms and TMD (HR $=1.7$ and odds ratio $[\mathrm{OR}]=3.6$, respectively), with prospective cohort evidence finding that OSA symptoms preceded firstonset TMD.

We found an increased risk of FP with smoking. Men who currently smoked cigarettes were at increased risk of reporting orofacial pain [4], but the results from a case-control study of TMD patients [6] did not show an association with smoking in either sex. It should be noted however that these studies did not take into account past smoking history or the duration or frequency of smoking. Marbach et al. [27] found that TMD cases were more likely to smoke, but no statistically significant difference was found for "ever smoking" or amount of cigarettes smoked per day. Smoking was reported to be associated with orofacial pain in a large study conducted in Australia [9]. Current tobacco users (both smoking and smokeless) were at increased risk of experiencing a range of painful oral symptoms [33] and the risk for oral pain decreased significantly if tobacco cessation occurred. It is likely that people who reported smoking are those with a less healthy life style. It can also be suggested that people with high levels of psychological distress are more likely to smoke. People with the highest quartile of psychological distress score were more likely to smoke, and this was true for both men and women [2]. In addition, smoking can also be thought to act directly as a mechanical factor. Often having a cigarette or pipe in the mouth may influence changes in joints and muscles in the face. Smokers also may be more likely to use chewing gum to disguise the tobacco smell and taste in the mouth. For example, smokers were twice as likely to use chewing gum and this was true for both men and women [4]. The other possible explanation is that smoking is a marker for some other unknown risk factors.

The reason for protective effect of alcohol is unclear. Recent study of self-reported alcohol consumption in 946 patients with fibromyalgia [34] showed that low and moderate alcohol consumption was associated with lower fibromyalgia symptoms and better QOL compared to no alcohol consumption.
Our analysis showed an increase in risk of FP by dental factors. Participants in a cross-sectional study conducted in the UK were more likely to report orofacial pain if they had more than five teeth missing [4]. A prospective study from Taiwan [35] compared a cohort of patients with chronic periodontitis and a cohort without a history of chronic periodontitis. Increased risk of developing trigeminal neuralgia during the 5-year follow-up period was higher in subjects with chronic periodontitis $(\mathrm{HR}=1.7)$ compared to control cohort but not among participants who had undergone a gingivectomy or periodontal flap operation.

The reporting of other bodily pain, such as upper body, leg pain and back pain, was also influential in the reporting of orofacial pain $[\underline{3}, \underline{7}, \underline{26}, \underline{30}]$. These findings confirm the results of a cohort study [28] where people with no TMD at baseline but at least one other pain condition were almost four times more likely to develop TMD in three years' time. It is likely that there are some areas of shared risk exposure for all these pain conditions. This suggests that the orofacial area may be just one body region involved in a more widespread musculoskeletal syndrome occurring as a consequence of common aetiological factors. It may also be one feature in a wider process of somatization.

\section{CONCLUSIONS}

This is the largest ever study to provide estimates of facial pain prevalence. It demonstrates unique features (lower prevalence than previously reported) and common features (more common in women) and confirms multifactorial aetiology of facial pain. Significant association with psychological distress and a strong relationship to pain elsewhere in the body suggests that aetiology is not specific to this regional pain.

\section{ACKNOWLEDGMENTS AND DISCLOSURE STATEMENTS}

This research has been conducted using the UK Biobank resource.

The authors report no conflicts of interest related to this study. 


\section{REFERENCES}

1. Macfarlane TV, Blinkhorn AS, Davies RM, Kincey J, Worthington HV. Oro-facial pain in the community: prevalence and associated impact. Community Dent Oral Epidemiol. 2002 Feb;30(1):52-60. [Medline: 11918576] [doi: 10.1034/j.1600-0528.2002.300108.x]

2. Macfarlane TV, Kincey J, Worthington HV. The association between psychological factors and oro-facial pain: a community-based study. Eur J Pain. 2002;6(6):427-34. [Medline: 12413431] [doi: 10.1016/S1090-3801(02)00045-9]

3. Macfarlane TV, Blinkhorn AS, Davies RM, Ryan P, Worthington HV, Macfarlane GJ. Orofacial pain: just another chronic pain? Results from a population-based survey. Pain. 2002 Oct;99(3):453-8. [Medline: 12406520] [doi: 10.1016/S0304-3959(02)00181-1]

4. Macfarlane TV, Blinkhorn AS, Davies RM, Worthington HV. Association between local mechanical factors and orofacial pain: survey in the community. J Dent. 2003 Nov;31(8):535-42. [Medline: 14554070] [doi: 10.1016/S0300-5712(03)00108-8]

5. Macfarlane TV, Glenny AM, Worthington HV. Systematic review of population-based epidemiological studies of orofacial pain. J Dent. 2001 Sep;29(7):451-67. [Medline: 11809323] [doi: 10.1016/S0300-5712(01)00041-0]

6. Macfarlane TV, Gray RJM, Kincey J, Worthington HV. Factors associated with the temporomandibular disorder, pain dysfunction syndrome (PDS): Manchester case-control study. Oral Dis. 2001 Nov;7(6):321-30. [Medline: 11834094] [doi: $10.1034 / j .1601-0825.2001 .00758 . x$ ]

7. Macfarlane TV, Kenealy P, Kingdon HA, Mohlin B, Pilley JR, Mwangi CW, Hunter L, Richmond S, Shaw WC. Orofacial pain in young adults and associated childhood and adulthood factors: results of the population study, Wales, United Kingdom. Community Dent Oral Epidemiol. 2009 Oct;37(5):438-50. Epub 2009 Jul 14. [Medline: 19624699] [doi: $10.1111 / \mathrm{j} .1600-0528.2009 .00482 . x]$

8. Mobilio N, Casetta I, Cesnik E, Catapano S. Prevalence of self-reported symptoms related to temporomandibular disorders in an Italian population. J Oral Rehabil. 2011 Dec;38(12):884-90. Epub 2011 May 20. [Medline: 21595739] [doi: $10.1111 / j .1365-2842.2011 .02228 . x$ ]

9. Sanders AE, Slade GD. Gender modifies effect of perceived stress on orofacial pain symptoms: National Survey of Adult Oral Health. J Orofac Pain. 2011 Fall;25(4):317-26. [Medline: 22247927]

10. McMillan AS, Wong MC, Zheng J, Lam CL. Prevalence of orofacial pain and treatment seeking in Hong Kong Chinese. J Orofac Pain. 2006 Summer;20(3):218-25. [Medline: 16913431]

11. Leung WS, McMillan AS, Wong MC. Chronic orofacial pain in southern Chinese people: experience, associated disability, and help-seeking response. J Orofac Pain. 2008 Fall;22(4):323-30. [Medline: 19090405]

12. Lipton JA, Ship JA, Larach-Robinson D. Estimated prevalence and distribution of reported orofacial pain in the United States. J Am Dent Assoc. 1993 Oct;124(10):115-21. [Medline: 8409001]

13. Allen N, Sudlow C, Downey P, Peakman TC, Danesh J, Elliott P, Gallacher J, Green J, Matthews P, Pell J, Sprosen T, Collins R. UK Biobank: Current status and what it means for epidemiology. Health Policy and Technology 2012 Sep;1(3):123-6. [doi: 10.1016/j.hlpt.2012.07.003]

14. Classification of chronic pain. Descriptions of chronic pain syndromes and definitions of pain terms. Prepared by the International Association for the Study of Pain, Subcommittee on Taxonomy. Pain Suppl. 1986;3:S1-226. [Medline: 3461421$]$

15. Townsend P, Phillimore P, Beattie A. Health and deprivation: Inequality and the north. J R Coll Gen Pract. May 1988; 38(310): 234. [PMC free article: 1711427]

16. Office for National Statistics. 2011 Census, Population Estimates by single year of age and sex for Local Authorities in the United Kingdom. Crown. 2013 Jul. [URL: http://www.ons.gov.uk/ons/rel/census/2011-census/population-estimatesby-single-year-of-age-and-sex-for-local-authorities-in-the-united-kingdom/index.html]

17. StataCorp. 2013. Stata Statistical Software: Release 13. College Station, TX: StataCorp LP [URL: http://www.stata.com/stata13/]

18. UK Biobank. Protocol for a large-scale prospective epidemiological resource. Protocol No: UKBB-PROT-09-06 (Main Phase). 2007 Mar. [URL: http://www.ukbiobank.ac.uk/wp-content/uploads/2011/11/UK-Biobank-Protocol.pdf]

19. Giannakopoulos NN, Rammelsberg P, Eberhard L, Schmitter M. A new instrument for assessing the quality of studies on prevalence. Clin Oral Investig. 2012 Jun;16(3):781-8. Epub 2011 May 19. [Medline: 21594656] [doi: 10.1007/s00784-011-0557-4]

20. Locker D, Grushka M. Prevalence of oral and facial pain and discomfort: preliminary results of a mail survey. Community Dent Oral Epidemiol. 1987 Jun;15(3):169-72. [Medline: 3474103] [doi: 10.1111/j.1600-0528.1987.tb00508.x]

21. Locker D, Miller Y. Subjectively reported oral health status in an adult population. Community Dent Oral Epidemiol. 1994 Dec;22(6):425-30. [Medline: 7882657] [doi: 10.1111/j.1600-0528.1994.tb00791.x]

22. Von Korff M, Dworkin SF, Le Resche L, Kruger A. An epidemiologic comparison of pain complaints. Pain. 1988 Feb;32(2):173-83. [Medline: 3362555] [doi: 10.1016/0304-3959(88)90066-8]

23. Goulet JP, Lavigne GJ, Lund JP. Jaw pain prevalence among French-speaking Canadians in Québec and related symptoms of temporomandibular disorders. J Dent Res. 1995 Nov;74(11):1738-44. [Medline: 8530734] [doi: $10.1177 / 00220345950740110401]$ 
24. Agerberg G, Bergenholtz A. Craniomandibular disorders in adult populations of West Bothnia, Sweden. Acta Odontol Scand. 1989 Jun;47(3):129-40. [Medline: 2756818] [doi: 10.3109/00016358909007693]

25. Riley JL 3rd, Gilbert GH, Heft MW. Socioeconomic and demographic disparities in symptoms of orofacial pain. J Public Health Dent. 2003 Summer;63(3):166-73. [Medline: 12962470] [doi: 10.1111/j.1752-7325.2003.tb03495.x]

26. Sipilä K, Ylöstalo PV, Joukamaa M, Knuuttila ML. Comorbidity between facial pain, widespread pain, and depressive symptoms in young adults. J Orofac Pain. 2006 Winter;20(1):24-30. [Medline: 16483017]

27. Marbach JJ, Lennon MC, Dohrenwend BP. Candidate risk factors for temporomandibular pain and dysfunction syndrome: psychosocial, health behavior, physical illness and injury. Pain. 1988 Aug;34(2):139-51. [Medline: 3174153 ] [doi: 10.1016/0304-3959(88)90159-5]

28. Von Korff M, Le Resche L, Dworkin SF. First onset of common pain symptoms: a prospective study of depression as a risk factor. Pain. 1993 Nov;55(2):251-8. [Medline: 8309712] [doi: 10.1016/0304-3959(93)90154-H]

29. Liao CH, Chang CS, Chang SN, Lane HY, Lyu SY, Morisky DE, Sung FC. The risk of temporomandibular disorder in patients with depression: a population-based cohort study. Community Dent Oral Epidemiol. 2011 Dec;39(6):525-31. Epub 2011 May 30. [Medline: 21623863] [doi: 10.1111/j.1600-0528.2011.00621.x]

30. Macfarlane TV, Worthington HV. Association between orofacial pain and other symptoms: a population-based study. Oral Biosci Med 2004;1:45-54. [URL: http://obm.quintessenz.de/obm_2004_01_s0045.pdf]

31. Smythe HA, Moldofsky H. Two contributions to understanding of the "fibrositis" syndrome. Bull Rheum Dis. 19771978;28(1):928-31. [Medline: 199304]

32. Sanders AE, Essick GK, Fillingim R, Knott C, Ohrbach R, Greenspan JD, Diatchenko L, Maixner W, Dubner R, Bair E, Miller VE, Slade GD. Sleep apnea symptoms and risk of temporomandibular disorder: OPPERA cohort. J Dent Res. 2013 Jul;92(7 Suppl):70S-7S. Epub 2013 May 20. [Medline: 23690360] [PMC free article: 3706181] [doi: $10.1177 / 0022034513488140]$

33. Leino P, Magni G. Depressive and distress symptoms as predictors of low back pain, neck-shoulder pain, and other musculoskeletal morbidity: a 10-year follow-up of metal industry employees. Pain. 1993 Apr;53(1):89-94.

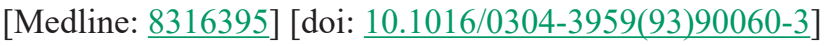

34. Kim CH, Vincent A, Clauw DJ, Luedtke CA, Thompson JM, Schneekloth TD, Oh TH. Association between alcohol consumption and symptom severity and quality of life in patients with fibromyalgia. Arthritis Res Ther. 2013 Mar 15;15(2):R42. [Medline: 23497427] [PMC free article: 3672769] [doi: 10.1186/ar4200]

35. Keller JJ, Sheu JJ, Lin HC. Chronic periodontitis and the subsequent risk of trigeminal neuralgia: a 5-year follow-up study. J Clin Periodontol. 2012 Nov;39(11):1017-23. Epub 2012 Sep 11. [Medline: 22966749] [doi: 10.1111/jcpe.12003]

\section{To cite this article:}

Macfarlane TV, Beasley M, Macfarlane GJ. Self-Reported Facial Pain in UK Biobank Study: Prevalence and Associated Factors.

J Oral Maxillofac Res 2014;5(3):e2

URL: http://www.ejomr.org/JOMR/archives/2014/3/e2/v5n3e2ht.pdf

doi: $10.5037 /$ jomr.2014.5302

Copyright (C) Macfarlane TV, Beasley M, Macfarlane GJ. Published in the JOURNAL OF ORAL \& MAXILLOFACIAL RESEARCH (http://www.ejomr.org), 1 October 2014.

This is an open-access article, first published in the JOURNAL OF ORAL \& MAXILLOFACIAL RESEARCH, distributed under the terms of the Creative Commons Attribution-Noncommercial-No Derivative Works 3.0 Unported License, which permits unrestricted non-commercial use, distribution, and reproduction in any medium, provided the original work and is properly cited. The copyright, license information and link to the original publication on (http://www.ejomr.org) must be included. 